\title{
An Improved Content-Aware Image Retargeting Method
}

\author{
Canlin Li, Xianbo Zheng and Shengnan Zhai \\ School of Computer and Communication Engineering, Zhengzhou University of \\ Light Industry, Zhengzhou 450000, China \\ lcl_zju@aliyun.com
}

\begin{abstract}
Traditional image resizing techniques are oblivious to the content of the image when changing its aspect ratio. In contrast, content-aware image retargeting is based on the analysis of content and it changes the size of an image through keeping the important features intact and removing unimportant regions. Seam carving is a classical algorithm based on content-aware. It used gradient magnitude to calculate the energy map, which can only detect the edge information of an image, so it will cause the visual prominent object deforming when doing image resizing if its internal texture is not so rich. In this paper, we propose a method of using the image gradient map and saliency map to create importance map, so that the edge structure information and the visual prominent object can be detected, and the visual prominent object will be maintained while resizing.
\end{abstract}

Key words: content-aware; gradient map; saliency map; seam carving

\section{Introduction}

With the development of the Internet and the popularity of mobile devices, visual media plays an increasingly important role in people's life. To accommodate high definition TVs, computer screens, PDAs and cell phones, the resolution or the aspect ratio of the image often must be adjusted [1]. If the resolution of the original image is not matched with the proportion of the target screen size, the image generated by the direct tension or the image of the adapted target screen size is distorted [2], and the visual saliency regions of the original image will generate obvious deformation. Geometric transformation is traditional image resizing technique, such as nearest neighbor, bilinear interpolation and bicubic interpolation. These methods often cannot result in satisfactory result because they are oblivious to the content of the image.

At present, in addition to the early resizing technique, there are uniform scaling and cropping. Standard image scaling is not sufficient since it is oblivious to the image content and typically can be applied only uniformly. Cropping is to cut the image into the same size as the display device, which will lose some of the important content of the original image and a large number of meaningful background information. The traditional image retargeting method can get the satisfactory target image only in the case of the same image aspect ratio as the original image. Once the original aspect ratio is changed, the visual object in the image can be squeezed or stretched. Traditional image retargeting methods often cannot result in satisfactory image because they are oblivious to the content of the image [3]. More effective retargeting methods can only be achieved by considering the image content and not only geometric constraints.

Seam carving [4] algorithm is the most classical content-aware image retargeting method. Compared with the traditional image resizing methods, it has achieved satisfactory results. However, after resizing an image through this algorithm the main areas of the image will be often deformed because of the limitation of only using the image gradient in energy function defining. For example, it will cause the visual prominent object deforming after resizing if the internal texture of the original image is 
not so rich. In order to solve the above problems, this paper uses the image gradient map and the saliency map to create importance map, and introduce the forward energy criterion at the same time. The importance map can be more complete to detect the image of the visual object, the important content will be maintained while resizing.

\section{Related Work}

Currently, content-aware image retargeting method is mainly based on the following three categories: Seam Carving, Wraping and Multi-operator.

The image operator seam carving is proposed by Avidan and Shamir, in which the least perceivable vertical seam or horizontal seam in the image is removed one by one to generate desired small-size images. Seam Carving is the most classic content-aware image retargeting method, which first proposed the energy function to describe the importance of image content. In an image, a seam is an optimal 8-connected path of low energy pixels crossing the image from top to bottom (or from left to right). The optimal seam can be found using dynamic programming. By repeatedly removing or inserting seams in one direction we can change the aspect ratio of an image, which the important regional content will be maintained while resizing.

Wraping is an efficient method to solve the problem of content-ware image retargeting, and the wraping function is generally obtained by a global optimization that scales or stretches homogeneous regions to minimize the resulting distortion. Liu and Gleicher[5] find the ROI and construct a novel Fisheye-View warp that essentially applies a piecewise linear scaling function in each dimension to the image. The method highlights and emphasizes important content while still maintaining the unimportant region. Shi Jian et al proposed a method of image retargeting based on triangular mesh parameterization, which was used to solve the mesh of the target image with constraint, and finally the target image was generated by the texture mapping. Wang et al[6] present a scale-and-stretch warping method. The method partitions the image into a grid mesh and deforms it into an arbitrary shape. Important image regions are optimized to scale uniformly while regions with homogeneous content are allowed to distort. However, since the distortion is distributed in all spatial directions, some objects may be excessively distorted, damaging the global spatial structure of the original image.

In 2009, Rubinstein et al[7] proposed an image retargeting method based on multi-operator, and their approach is supported by a user's study they conducted that clearly shows that users generally prefer to use more than one operator to achieve better results. They propose an algorithm for resizing space to find the optimal path, using a new global bidirectional warping function for evaluating the retargeting results. Dong weiming et al [8] also combines seam carving and scaling in this order to obtain the final result. An image distance function formulated as a combination of a patch-based bidirectional image Euclidean distance, dominant color descriptor (DCD) similarity, and seam energy variation is proposed. The method is very effective for maintaining the important region and the background information of the image.

Compared with the above image retargeting methods, the seam carving method can overcome the ratio distortion caused by the non-proportional scaling, and can maintain the image structure in a certain degree. However, since it uses the gradient of the image to define the energy function and the gradient can only detect the edge of the image structure information, the method has some limitations. For example, it will cause the visual prominent object deforming when doing image resizing if the internal texture of image is not so rich, thus affecting the integrity of the image structure information. 


\section{Algorithm Description}

Seam carving is an algorithm based on image content-aware. The algorithm uses the importance map to determine the important areas of the image. The method uses the image gradient as the importance map, using a dynamic programming algorithm to find the energy minimum seam path, by iteratively insert or remove optimal seam to change the aspect ratio of the image. The algorithm defines seam as the connected path of low energy pixels crossing the image from top to bottom (or from left to right). Seam carving is based on the definition of an energy function defining the importance of pixels. In this paper, we propose a method of using the image gradient map and saliency map to create importance map, so that the edge structure information and the visual prominent object can be detected. In order to improve the energy function of the carving seam algorithm, it can effectively avoid the seam through the image salient region, and the result is more satisfactory. Algorithm flow shown in Figure 1:

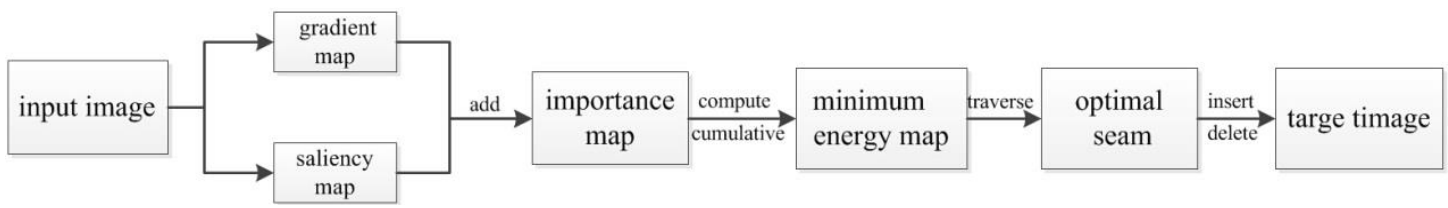

Figure 1. The Work Flow Chart of the Algorithm Proposed in this Paper

\subsection{Define the Energy Function}

The basic idea of the seam carving algorithm is to change the aspect ratio of the image by iteratively removing or inserting the seam of cumulative minimum energy. The importance of image pixel is defined by the energy function, the energy value of pixel is greater, the higher degree of attention to the visual. On the contrary, the energy value is smaller, less attention to. Therefore, importance map of image is important for image retargeting.

Since human vision is more sensitive to edges, Avidan and Shamir give high value to edges using the following simple energy function. They used image gradient algorithm to define energy function [3]. Set an image as I, and its size is $m \times n$, the gradient function of the image is:

$$
e(I)=\left|\frac{\partial}{\partial_{x}} I\right|+\left|\frac{\partial}{\partial_{y}} I\right|
$$

Sobel operator is a discrete first order differential operator, which has the advantages of simple calculation, high speed and smooth effect on noise and the edge information is more accurate. Therefore, the traditional seam carving method uses sobel edge detection algorithm to generate the gradient map as importance map of the image. However, the gradient map can only detect the image edge structure information. If the edge information of the image is scattered, the region of interest with a lower degree of importance will be wrongly identified as background information area, and the important areas of the image will be a certain degree of deformation after resizing.

\subsection{Improved Energy Function}

For the shortcomings of traditional seam carving algorithm, the researchers used visual saliency map to measure the content of the image visual importance, which corresponds to the important content of the image to be protected. Saliency detection method is divided into the following two categories: one is based on low-level visual information by data driven from the bottom-up attention model [9]; the other is driven by tasks and 
knowledge of the top-down high-level visual attention model. The saliency detection method in this paper is based on the low level visual information, which is based on the full resolution model algorithm (Frequency-tuned) proposed by Achanta et al[10]. The method can quickly calculate the visual saliency map by using the color and brightness information of the image, and get the clear boundary information while maintaining the integrity of the visual saliency object [11].

A content-aware retargeting operator relies on an importance map to preserve the important parts of the media at the expense of the less-important ones. Importance measures include image gradients, saliency and entropy, as well as high level cues such as face detectors. In this paper, we propose a method that combines image gradient map and saliency map to generate an importance map. The new energy function is defined as:

$E_{\text {new }}(i, j)=w_{g} e(i, j)+w_{s} E_{s}(i, j)$

(2)

where $e(i, j)$ is the image gradient map, and $E_{s}(i, j)$ is the image saliency map. Where $w_{g}$ and $w_{s}$ are the weight parameters. To determine the two weights, a couple of experiments are needed. In this paper, we set $w_{g}=0.5$, and $w_{s}=0.5$, which are used to adjust the image gradient and the importance of visual saliency in the whole energy map. As shown in Figure 2, this paper uses the Sobel algorithm and the FT algorithm[10] to generate the importance map, it can be clearly seen that the edge information and the visual subject are more complete after the weighted energy map.

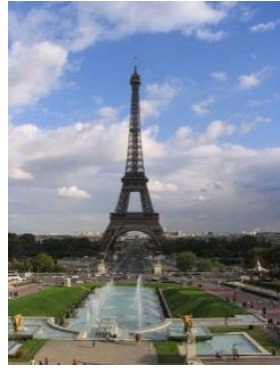

(a) Original

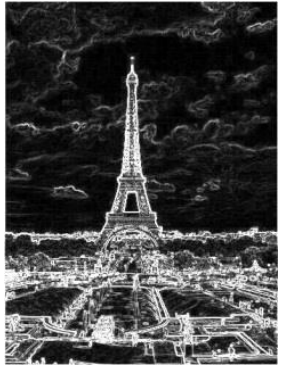

(b) Sobel

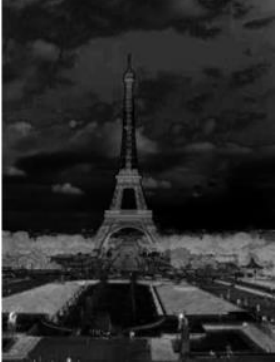

(c) the method in this paper

\section{Figure 2. Importance Maps Respectively Generated by Sobel and the Algorithm in this Paper}

\subsection{Find the Optimal Seam}

Formally, let I be an $m \times n$ image, the term vertical seam[4] is defined as:

$S^{x}=\left\{s_{i}^{x}\right\}_{i=1}^{n}=\{(x(i), i)\}_{i=1}^{n}, \forall i,|x(i)-x(i-1)| \leq 1$

Where $x$ is a mapping $x:[1, \ldots, n] \rightarrow[1, \ldots, m]$ that is continuous in a discrete sense. Similarly, if $y$ is a mapping $y:[1, \ldots, m] \rightarrow[1, \ldots, n]$, then a horizontal seam is defined to be:

$S^{y}=\left\{s_{y}^{m}\right\}_{j=1}^{m}=\{(j, y(j))\}_{j=1}^{m}, \forall j,|y(j)-y(j-1)| \leq 1$

(4)

A seam is an 8-connected path of low energy pixels in the image from top to bottom, containing one, and only one, pixel in each row of the image. All pixels on a seam can be expressed as (vertical seam as an example) $I_{s}=\left\{I\left(s_{i}\right)\right\}_{i=1}^{n}=\{I(x(i), i)\}_{i=1}^{n}$. Remove a seam similar to the removal of a row or column from an image, all the pixels of the image 
are shifted left (or up) to compensate for the missing path [12]. The image changes only at the seam region, while the other areas remain intact.

The seam energy will be the sum of the energy of pixels constituting the seam. We can define the cost of a seam as $E(s)=E\left(I_{s}\right)=\sum_{i=1}^{n} E\left(I\left(s_{i}\right)\right)$, therefore the optimal seam $s *$ is the minimum energy cost:

$s^{*}=\min _{s} E(s)=\min _{s} \sum_{i=1}^{n} E\left(I\left(s_{i}\right)\right)$

In the following, guided by the energy map, minimum energy seam can be found by dynamic programming algorithm. The first step is to traverse the image from the second row to the last row and calculate the cumulative minimum energy $M(i, j)$ for all the possible seams on each entry $(i, j)$ :

$M(i, j)=E_{\text {new }}(i, j)+\min (M(i-1, j-1), M(i-1, j), M(i-1, j+1))$

In the second step we backtrack from this minimum entry on $M$ to find the path of the optimal seam. The method only considers the energy to remove the pixel itself, ignoring the newly inserted energy. The inserted energy is due to new intensity edges created by previously nonadjacent pixels that become neighbors once the seam is removed, for this reason we introduce the forward energy criterion[13]. According to the connectivity of the seam, there are three cases of adjacent pixels in the vertical seam. For each of the three possible cases, we define a cost respectively:

$$
\begin{aligned}
& C_{L}(i, j)=|I(i, j+1)-I(i, j-1)|+|I(i-1, j)-I(i, j-1)| \\
& C_{u}(i, j)=|I(i, j+1)-I(i, j-1)| \\
& C_{R}(i, j)=|I(i, j+1)-I(i, j-1)|+|I(i-1, j)-I(i, j+1)|
\end{aligned}
$$

For vertical seams, each cost $M(i, j)$ is updated using the following rule[13]:

$M(i, j)=E_{\text {new }}(i, j)+\min \left\{\begin{array}{c}M F(i-1, j-1)+C_{L}(i, j) \\ M F(i-1, j)+C_{u}(i, j) \\ M F(i-1, j+1)+C_{R}(i, j)\end{array}\right.$

In this paper, the proposed algorithm introduces the forward energy criterion, and the dynamic programming algorithm[14] is used to find the minimum cumulative energy seam by backtracking method, which can change the size of the image. In this way, the improved seam carving with forward energy criterion can effectively remove the artificial noise generated by method addressed in traditional algorithm. 
4. Experimental Analysis and Comparison

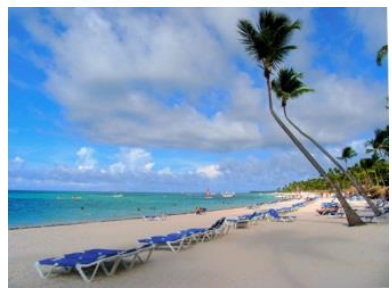

(a) Original image

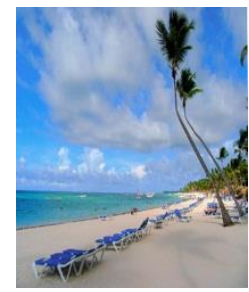

(b) Scaling

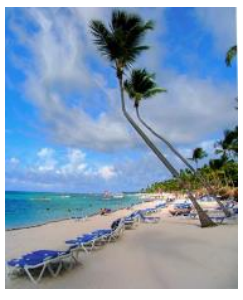

(c) OSS

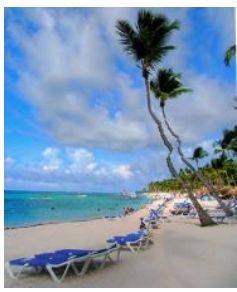

(d) Seam carving

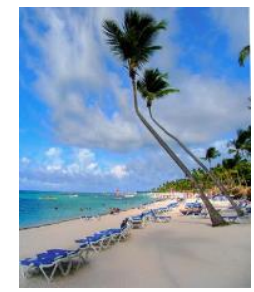

(e) Ours

Figure 3. Comparison of our Results with those of Scaling, OSS and Seam Carving. The original Image is Retargeted to about $75 \%$ Width

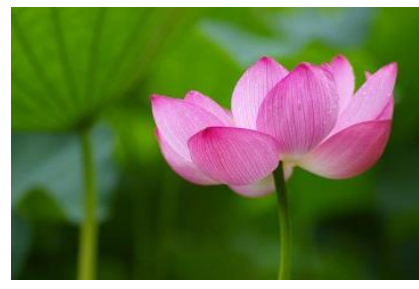

(a) Original image

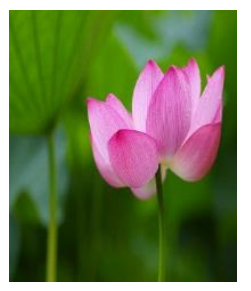

(b) Scaling

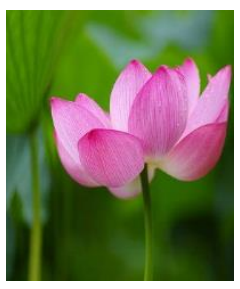

(c) OSS

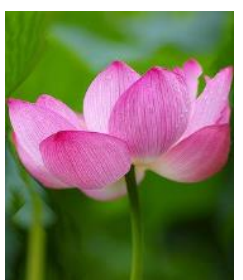

(d) Seam carving

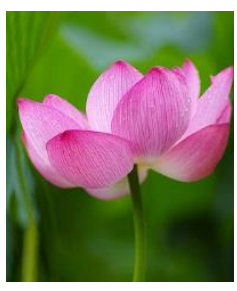

(e) Ours

Figure 4. Comparison of our Results with those of Scaling, OSS and Seam Carving. The Original Image is Retargeted to about $75 \%$ Width

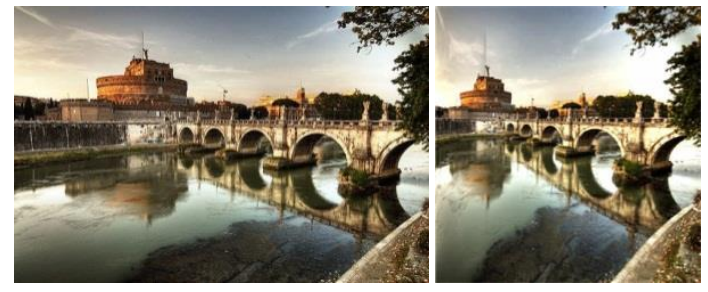

(a) Original image

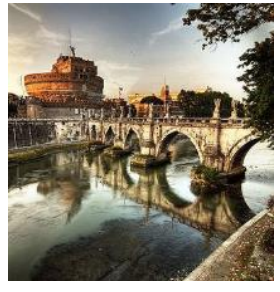

(c) OSS

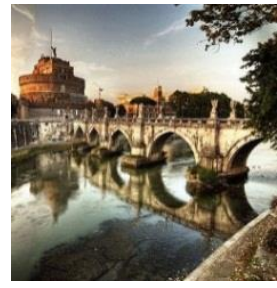

(d) Seam carving

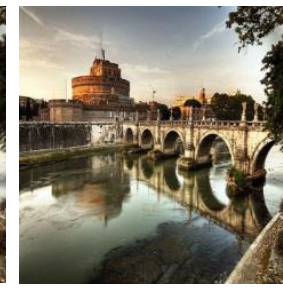

(e) Ours

Figure 5. Comparison of our Results with those of Scaling, OSS and Seam Carving. The Original Image is Retargeted to about $75 \%$ Width

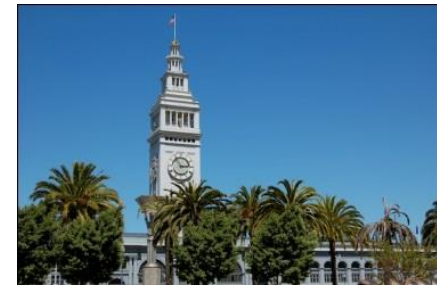

(a) Original image

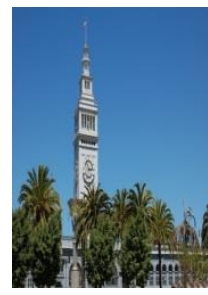

(b) Scaling

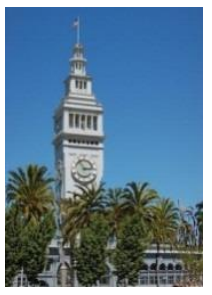

(c) OSS

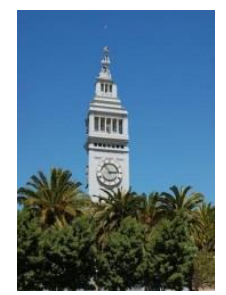

(d) Seam carving

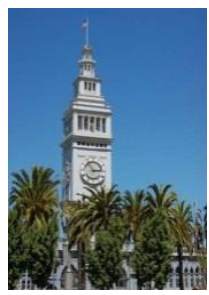

(e) Ours

Figure 6. Comparison of Different Retargeting Operator. The Original Image is Retargeted to about $50 \%$ Width 


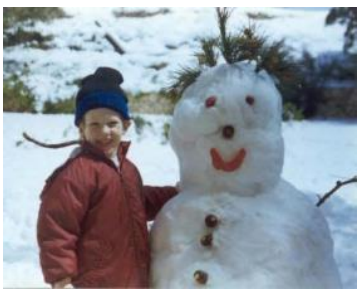

(a) Original image

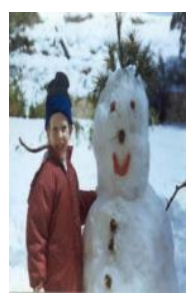

(b) Scaling

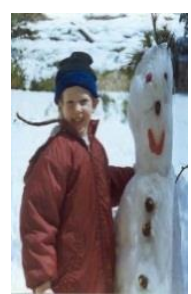

(c) OSS

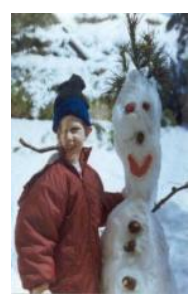

(d) Seam carving

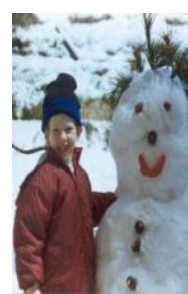

(e) Ours

Figure 7. Comparison of different Retargeting Operator. The Original Image is Retargeted to about $50 \%$ Width

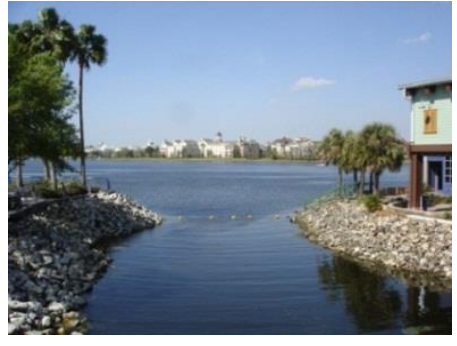

(a) Original image

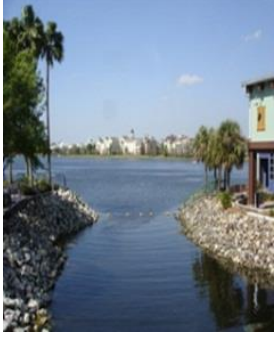

(b) Scaling

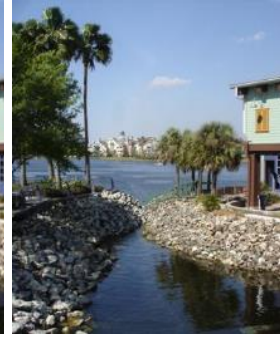

(c) OSS

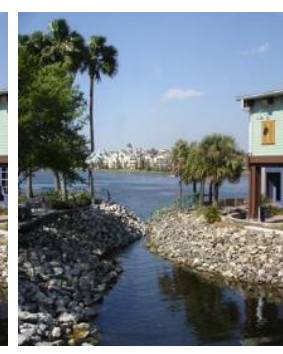

(d) Seam carving

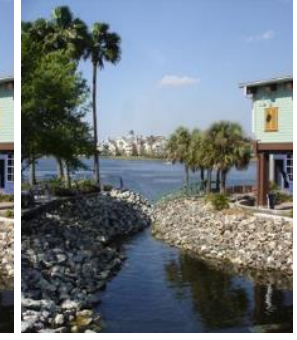

(e) Ours

Figure 8. Comparison of Different Retargeting Operator. The Original Image is Retargeted to about $\mathbf{5 0 \%}$ Width

In this paper, the improved seam carving algorithm is compared with scaling, optimized scale-and-stretch (oss) and traditional seam carving algorithm. In Figure 3, Figure 4 and Figure 5 the original image is retargeted to about $75 \%$ width. As shown in Figure 3 the trees of the traditional seam carving algorithm appear broken, the trees and chairs of the improved algorithm to keep the overall structure. In Figure 4, we can see that the image in our result preserves the global visual effect better. The algorithm remains lotus shape intact, and there is less deformation. In Figure 5, we can see that the proportion of the overall structure of the building is changed in the uniform scaling algorithm. In Figure 6, Figure 7 and Figure 8 the original image is retargeted to about $50 \%$ width. As shown in Figure 5, Figure 6 and Figure 8, we find that our algorithm is feasible for resizing the width of the image, especially when the resizing proportions are different. We can also see that our measure is better in preserving the aspect ratio of some objects, for example the child and the snowman in Figure 7. In Figure 8, we show the efficiency of our algorithm. In the result by our method, trees and houses are not deformed.

\section{Conclusions}

In order to overcome the shortcomings of the traditional seam carving algorithm, this paper proposes a method of using the image gradient map and saliency map to define the energy function. According to the importance map of the image, the proposed method removes or inserts a low energy seam, so as to avoid the seam pass through important regions in the image. Experimental results show that the improved method in this paper gets better results than the traditional seam carving algorithm. The problem is that the algorithm is highly dependent on the importance map. The focus of the work in the future is to improve the calculation method of the importance map, such as add the face detection to the energy function to enhance the protection of human face while resizing. In addition to the arbitrary resizing of images, our method has potential use in video resizing. 


\section{References}

[1] Chen, L., Xie, X., Fan, X., Ma, W., Zhang, H., and Zhou, H. A visual attention model for adapting images on small displays. Multimedia Systems, 2003. 9(4): p. 353-364.

[2] Fun, L., Xie, X., Ma, W., Zhang, H., and Zhou, H. Visual attention based image browsing on mobile devices, in Proceedings-IEEE International Conference on Multimedia and Expo. 2003. p. 53-56.

[3] Avidan, S. and A. Shamir, Seam carving for content-aware image resizing. ACM Transactions on Graphics, 2007. 26(99): p. 10.

[4] Shamir, A. and S. Avidan, Seam carving for media retargeting. Communications of the ACM, 2009. 52(1): p. 77.

[5] Liu, F. and M. Gleicher, Automatic Image Retargeting with Fisheye-View Warping. Proceedings of the Annual ACM Symposium on User Interface Softaware and Technology, 2005: p. 153-162.

[6] Wang, Y.-S., Tai, C.-L., Sorkine, O. and Lee, T.-Y. Optimized scale-and-stretch for image resizing. ACM Transactions on Graphics, 2008. 27(5): p. 1.

[7] Rubinstein, M., A. Shamir and S. Avidan, Multi-operator media retargeting. ACM Transactions on Graphics, 2009. 28(3): p. 1.

[8] Dong, W., Zhou, N., Paul, J.-C., and X. Zhang, Optimized image resizing using seam carving and scaling. ACM Transactions on Graphics, 2009. 28(5): p. 1.

[9] Itti, L., C. Koch and E. Niebur, A Model of Saliency-Based Visual Attention for Rapid Scene Analysis. IEEE Transactions on Pattern Analysis and Machine Intelligence, 1998. 11(20): p. 1254-1259.

[10] Achanta, R., Hemami, S., Estrada, F. and S. Susstrunk, Frequency-tuned salient region detection, in IEEE International Conference on Computer Vision and Pattern Recognition (CVPR). 2009. p. 1597-1604.

[11] Achanta, R. and S. Susstrunk, Saliency detection for content-aware image resizing, in 2009 IEEE International Conference on Image Processing. 2009. p. 1005-1008.

[12] Yan, Z. and H. Chen, A Study of Image Retargeting Based on Seam Carving, in Proceedings - 2014 6th International Conference on Measuring Technology and Mechatronics Automation. 2014. p. 60-63.

[13] Rubinstein, M., A. Shamir and S. Avidan, Improved seam carving for video retargeting. ACM Transactions on Graphics, 2008. 27(3): p. 1.

[14] Dong, W., Zhou, N., Paul, J.-C., and X. Zhang, Fast Multi-Operator Image Resizing and Evaluation. Journal of Computer Science and Technology, 2012. 27(1): p. 121-134.

\section{Authors}

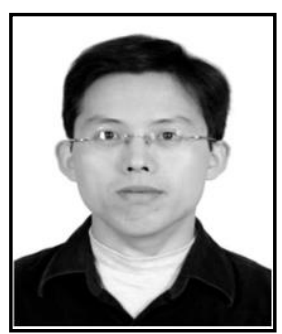

Li Canlin, He received $\mathrm{PhD}$ degree in computer science from Shanghai Jiaotong University in 2010. Since 2010 he has been in the School of Computer and Communication Engineering at Zhengzhou University of Light Industry. His research interests include image processing, multimedia, graphics, digital entertainment and software engineering. 